\title{
SUR LA RÉFLEXIVITÉ DES PRODUITS TENSORIELS ET LES SOUS-ESPACES DES PRODUITS TENSORIELS PROJECTIFS
}

\author{
EVE OJA*
}

\section{Résumé.}

Soient $X$ et $Y$ deux espaces de Banach. Nous caractérisons la réflexivité de $X \hat{\otimes} Y$ et de $X \hat{\otimes} Y$ dans le cas où $X$ possède une décomposition inconditionnelle de dimension finie; nous étudions des propriétés de reproductibilité des espaces $l_{q}$ dans $l_{p} \hat{\otimes} Y$ et caractérisons les sous-espaces de $l_{p} \hat{\otimes} l_{r}$ isomorphes à $l_{q}$.

\section{Introduction.}

Soient $X$ et $Y$ deux espaces de Banach dont les duaux respectifs sont notés $X^{\prime}$ et $Y^{\prime}$. La norme $\varepsilon$ sur le produit tensoriel $X \otimes Y$ est définie par

$$
\|u\|_{\varepsilon}=\sup \left\{|(f \otimes g)(u)|: f \in X^{\prime},\|f\| \leqq 1, g \in Y^{\prime},\|g\| \leqq 1\right\}
$$

et la norme $\pi$ est définie par

$$
\|u\|_{\pi}=\inf \left\{\sum_{i=1}^{n}\left\|x_{i}\right\|\left\|y_{i}\right\|: u=\sum_{i=1}^{n} x_{i} \otimes y_{i}\right\} .
$$

Notons $X \otimes_{\varepsilon} Y$ (respectivement $X \otimes_{\pi} Y$ ) le produit tensoriel $X \otimes Y$ muni de la norme $\varepsilon$ (respectivement $\pi$ ) et $X \hat{\otimes} Y$ (respectivement $X \hat{\otimes} Y$ ) le complété de $X \otimes_{\varepsilon} Y$ (respectivement $X \otimes_{\pi} Y$ ).

Rappelons qu'une décomposition de Schauder d'un espace de Banach $X$ est une suite $\left(p_{k}\right)$ de projections non nulles, continues de $X$ qui satisfont aux conditions: $p_{k} \circ p_{l}=0$ si $k \neq l$ et pour tout $x \in X$ la série $\sum_{k=1}^{\infty} p_{k} x$ converge vers $x$. Si la convergence est inconditionnelle pour tout $x \in X$ alors on dit que la décomposition de Schauder $\left(p_{k}\right)$ est inconditionnelle et si $\operatorname{dim} \operatorname{Im} p_{k}<\infty$ pour tout $k=1,2, \ldots$ alors on dit que $\left(p_{k}\right)$ est une décomposition de dimension finie.

Le symbole $l_{\infty}$ notera ici l'espace $c_{0}$ des suites de scalaires qui convergent

* Ce travail a été effectué durant un stage de l'auteur à l'Université Aix-Marseille II dirigé par les Professeurs P. Billard et C. Samuel auxquels nous adressons nos plus vifs remerciements.

Reçu le 29. juin 1981. 
vers 0 muni de la norme sup. Si $1 \leqq p \leqq \infty$ alors $p^{\prime}$ note le réel défini par $1 / p$ $+1 / p^{\prime}=1$ avec la convention $p^{\prime}=\infty$ si $p=1$ et $p^{\prime}=1$ si $p=\infty$.

Pour les définitions et propriétés classiques cf. [1] et [7].

Il existe une caractérisation "duale» de la réflexivité des produits tensoriels $X \hat{\otimes} Y$ et $X \hat{\otimes} Y$ pour des espaces de Banach réflexifs $X$ et $Y$ dont l'un possède la propriété d'approximation (qui découle du critère de réflexivité de l'espace des opérateurs compacts (cf. p. ex. [5]) et qui a été établie par J. R. Holub [3] pour $X$ et $Y$ ayant des bases): le produit tensoriel $X \hat{\hat{\otimes}} Y$ (respectivement $X \hat{\otimes} Y)$ est réflexif si et seulement si tout opérateur linéaire continu de $X^{\prime}$ dans $Y$ est compact (respectivement tout opérateur linéaire continu de $X$ dans $Y^{\prime}$ est compact).

En restreignant la classe des espaces réflexifs $X$ et $Y$ considérés nous avons, d'après N. J. Kalton [5], un autre critère de réflexivité de $X \hat{\hat{\otimes}} Y$ : si $X$ possède la propriété d'approximation bornée inconditionnelle (c.-à-d. s'il existe une suite $\left(A_{k}\right)$ d'opérateurs de rang fini de $X$ dans $X$ telle que pour tout $x \in X$ la série $\sum_{k=1}^{\infty} A_{k} x$ converge inconditionnellement vers $x$ ) alors le produit tensoriel

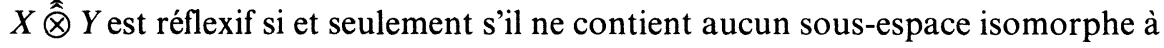
$c_{0}$. Bien que, dans ces conditions, nous ne connaissions pas de critère "dual » pour $X \hat{\otimes} Y$, nous donnerons ici une caractérisation "duale " de la réflexivité des produits tensoriels $X \hat{\otimes} Y$ et $X \hat{\otimes} Y$ pour des espaces réflexifs $X$ et $Y$ dont l'un possède une décomposition inconditionnelle de dimension finie. Nous montrerons (cf. Théorème 2) que dans ces conditions le produit tensoriel $X \hat{\hat{\otimes}} Y$ (respectivement $X \hat{\otimes} Y$ ) est réflexif si et seulement s'il ne contient aucun sousespace complémenté isomorphe à $c_{0}$ (respectivement $l_{1}$ ).

C. Samuel [11] a établi que si pour un espace de Banach $Y$ et $1 \leqq q<p \leqq \infty$ l'espace $l_{p} \hat{\hat{\otimes}} Y$ a un sous-espace isomorphe à $l_{q}$ alors $Y$ a un sous-espace isomorphe à $l_{q}$ et si pour $1 \leqq p<q \leqq \infty$ l'espace $l_{p} \hat{\otimes} Y$ a un sous-espace isomorphe à $l_{q}$ alors le bidual $Y^{\prime \prime}$ a un sous-espace isomorphe à $l_{q}$. Nous montrerons (cf. Théorème 3) que pour $l_{p} \hat{\otimes} Y$ la situation est analogue au cas de $l_{p} \hat{\otimes} Y$ : c'est $Y$ qui a un sous-espace isomorphe à $l_{q}$.

Pour $l_{p} \hat{\otimes} l_{r}, 1 \leqq p, r \leqq \infty$, nous obtiendrons les résultats "duaux » des résultats de C. Samuel (cf. [11] et [12]) sur $l_{p} \hat{\otimes} l_{r}$. Nous montrerons (cf. Théorème 4) que si $l_{q}$ est isomorphe à un sous-espace de $l_{p} \hat{\otimes} l_{r}$ alors $q=p$ ou $q=r$ ou $q=s$ où $s=1$ si $p \leqq r^{\prime}$ et $1 / s^{\prime}=1 / p+1 / r$ (avec $\left.1 / \infty=0\right)$ si $p>r^{\prime}$ et, d'autre part (cf. Théorème 5), tout sous-espace fermé de dimension infinie de $l_{p} \hat{\otimes} l_{r}$ contient un sous-espace complémenté dans $l_{b} \hat{\otimes} l_{r}$ et isomorphe à $l_{a}$ pour les mêmes valeurs de $q$. (Rappelons que dans le case de $l_{p} \hat{\hat{\otimes}} l_{r}$ on avait $s=\infty$ si $p \geqq r^{\prime}$ et $1 / s=1 / p+1 / r-1$ si $p<r^{\prime}$ (cf. [11] et [12]).)

La démonstration de ces résultats repose sur le fait que les produits tensoriels considérés ont une "bonne " décomposition de Schauder. 


\section{Décompositions de Schauder et réflexivité.}

Soient $X$ et $Y$ deux espaces de Banach et $X \hat{\otimes}_{\alpha} Y$ le complété du produit tensoriel $X \otimes_{\alpha} Y$ où $\alpha=\varepsilon$ ou $\alpha=\pi$. Soit $\left(\pi_{k}\right)$ une décomposition de Schauder de $X$ et $I_{Y}$ l'identité sur $Y$. Pour $k=1,2, \ldots$ notons $p_{k}$ le prolongement continu de

$$
\pi_{k} \otimes I_{Y}: X \otimes_{\alpha} Y \rightarrow X \otimes_{\alpha} Y
$$

à $X \hat{\otimes}_{\alpha} Y$. Il est immédiat de vérifier (cf. p. ex. [2]) que $\left(p_{k}\right)$ est une décomposition de Schauder de $X \hat{\otimes}_{\alpha} Y$ et si $\left(\pi_{k}\right)$ est inconditionnelle alors $\left(p_{k}\right)$ est aussi inconditionnelle. (Notons que le résultat reste valable si $\alpha$ est une « uniform crossnorm ».)

Remarquons que $\operatorname{Im} \pi_{k} \hat{\hat{\otimes}} Y$ est isométrique au sous-espace $\operatorname{Im} p_{k}$ de $X \hat{\otimes} Y$ (puisque la norme $\varepsilon$ sur $\operatorname{Im} \pi_{k} \otimes Y$ coïncide avec la norme induite par la norme $\varepsilon$ de $X \otimes Y$ ) et que $\operatorname{Im} \pi_{k} \hat{\otimes} Y$ est isomorphe au sous-espace $\operatorname{Im} p_{k}$ de $X \hat{\otimes} Y$ (pour la preuve il suffit de noter que $\operatorname{Im} \pi_{k} \otimes_{\pi} Y$ est isomorphe à un sous-espace de $X \otimes_{\pi} Y$ puisque $\operatorname{Im} \pi_{k}$ est complémenté dans $\left.X\right)$. Donc si $\left(\pi_{k}\right)$ est une décomposition de dimension finie, c.-à-d. si $d_{k}=\operatorname{dim} \operatorname{Im} \pi_{k}<\infty$ pour tout $k=1,2, \ldots$, alors $\operatorname{Im} p_{k}$ (comme sous-espace de $X \hat{\otimes} Y$ ou $X \hat{\otimes} Y$ ), isomorphe au produit normé $l_{1}^{d_{k}}(Y)$, est isomorphe au produit $Y^{d_{k}}$.

Rappelons qu'une décomposition de Schauder $\left(p_{k}\right)$ d'un espace de Banach $X$ est dite "shrinking» si $\left(p_{k}^{\prime}\right)$ est une décomposition de Schauder pour $X^{\prime}$ et $\left(p_{k}\right)$ est dite « boundedly complete " si pour toute suite bornée $\left(\sum_{k=1}^{n} x_{k}\right)_{n=1,2, \ldots}$, $x_{k} \in \operatorname{Im} p_{k}$, la série $\sum_{k=1}^{\infty} x_{k}$ est convergente.

La proposition suivante trouve son analogue dans [3] où $X$ et $Y$ étaient deux espaces avec des bases et où l'on considérait le produit tensoriel des bases.

Proposition 1. Soient $X$ et $Y$ deux espaces de Banach, $X$ possédant une décomposition de dimension finie $\left(\pi_{k}\right)$. Si $\left(\pi_{k}\right)$ est "shrinking " alors la décomposition de Schauder $\left(p_{k}\right)$ de $X \hat{\otimes} Y$ est "shrinking ". Si $\left(\pi_{k}\right)$ est " boundedly complete " alors la décomposition de Schauder $\left(p_{k}\right)$ de X $\hat{\otimes} Y$ est " boundedly complete".

Supposons $\left(\pi_{k}\right)$ " shrinking ". Comme $\left(\pi_{k}^{\prime}\right)$ est une décomposition de Schauder de $X^{\prime}$, les prolongements continus $q_{k}$ de $\pi_{k}^{\prime} \otimes I_{Y^{\prime}}$ forment une décomposition de Schauder de $X^{\prime} \hat{\otimes} Y^{\prime}$. D'autre part, $X^{\prime} \hat{\otimes} Y^{\prime}$ s'identifie canoniquement à $(X \hat{\otimes} Y)^{\prime}$ (puisque $X^{\prime}$ est séparable et posséde la propriété d'approximation) (cf. [1]) et $q_{k}=p_{k}^{\prime}$ par cette identification; donc ( $\left.p_{k}^{\prime}\right)$ est une décomposition de Schauder de $(X \hat{\hat{\otimes}} Y)^{\prime}$, c.-à-d. $\left(p_{k}\right)$ est "shrinking ".

Si $\left(\pi_{k}\right)$ est " boundedly complete " alors $X$ est canoniquement isomorphe à $H^{\prime}$ (où $H=\left\{f \in X^{\prime}: \lim _{n \rightarrow \infty}\left\|\sum_{k=1}^{n} \pi_{k}^{\prime} f-f\right\|=0\right\}$, muni de la topologie de norme) et $\left(\left.\pi_{k}^{\prime}\right|_{H}\right)$ est une décomposition de Schauder de $H$ qui est "shrinking" (cf. [10]). D'après ce que nous venons de démontrer, la décomposition de Schauder $\left(q_{k}\right)$ de $H \hat{\otimes} Y^{\prime}$ formée des prolongements continus de $\left.\pi_{k}^{\prime}\right|_{H} \otimes I_{Y^{\prime}}$ est 
" shrinking » et par conséquent la décomposition de Schauder $\left(q_{k}^{\prime}\right)$ de $\left(H \hat{\otimes} Y^{\prime}\right)^{\prime}$ est " boundedly complete » (cf. [4]). Mais $H^{\prime}$ étant séparable et possédant la propriété d'approximation, $\left(H \hat{\otimes} Y^{\prime}\right)^{\prime}$ s'identifie canoniquement à $H^{\prime} \hat{\otimes} Y^{\prime \prime}$ qui contient $H^{\prime} \hat{\otimes} Y$ comme sous-espace (cf. [1]). En utilisant la dualité naturelle $\left(X \otimes Y, X^{\prime} \otimes Y^{\prime}\right)$ il est facile de vérifier que par l'identification canonique $q_{k}^{\prime}$ prolonge $\pi_{k} \otimes I_{Y}$ et par conséquent les prolongements continus de $\pi_{k} \otimes I_{Y}$ forment une décomposition de Schauder " boundedly complete " pour $H^{\prime} \hat{\otimes} Y$, donc aussi pour $X \hat{\otimes} Y$.

Remarquons que la décomposition (inconditionnelle) de Schauder $\left(p_{k}\right)$ construite à partir de la décomposition de Schauder $\left(\pi_{k}\right)$ de $l_{2}$ associée naturellement à sa base canonique (notons que $\left(\pi_{k}\right)$ est de dimension finie, inconditionnelle, "shrinking " et " boundedly complete ") n'est pas " boundedly complete" pour $l_{2} \hat{\otimes} l_{2}$ et n'est pas «shrinking" pour $l_{2} \hat{\otimes} l_{2}$. En effet, comme $l_{2} \hat{\otimes} l_{2}$ contient un sous-espace isomorphe à $c_{0}$ et $l_{2} \hat{\otimes} l_{2}$ contient un sous-espace isomorphe à $l_{1}$ (cf. p. ex. [11]), il reste à appliquer la proposition suivante qui découle immédiatement du Corollaire $2 \mathrm{du}$ Théorème $2.4 \mathrm{de}[8]$ et du Corollaire du Théorème $1 \mathrm{de} \mathrm{[9]} \mathrm{(pour} \mathrm{le} \mathrm{(a)),} \mathrm{du}$ Théorème 2, (b), de [9] (pour le (b)) et du Corollaire 2 du Théorème 2.7 de [8] (pour le (c)).

Proposition 2. Soit $\left(p_{k}\right)$ une décomposition de Schauder inconditionnelle d'un espace de Banach $X$.

(a) $\mathrm{Si} \operatorname{Im} p_{k}$ est faiblement séquentiellement complet pour tout $k=1,2, \ldots$ alors les conditions suivantes sont équivalentes:

(i) $\left(p_{k}\right)$ est " boundedly complete ",

(ii) $X$ est faiblement séquentiellement complet,

(iii) $X$ ne contient aucun sous-espace complémenté isomorphe à $c_{0}$.

(b) Si $X$ ne contient aucun sous-espace complémenté isomorphe à $l_{1}$ alors $\left(p_{k}\right)$ est " shrinking ".

(c) Si le dual $\left(\operatorname{Im} p_{k}\right)^{\prime}$ est séparable pour tout $k=1,2, \ldots$ et $\left(p_{k}\right)$ est " shrinking " alors $X$ ne contient aucun sous-espace isomorphe $\dot{a} l_{1}$.

Notons que des propositions 1 et 2, (a), découle immédiatement un résultat de S. Heinrich [2]: si deux espaces de Banach $X$ et $Y$ sont faiblement séquentiellement complets et $X$ a une décomposition inconditionnelle de dimension finie alors $X \hat{\otimes} Y$ est faiblement séquentiellement complet. En effet, dans ce cas la décomposition $\left(\pi_{k}\right)$ de $X$ est " boundedly complete " et par conséquent la décomposition inconditionnelle $\left(p_{k}\right)$ de $X \hat{\otimes} Y$ est aussi "boundedly complete " ce qui est équivalent à la faible séquentielle complétion de $X \hat{\otimes} Y$ puisque l'image $\operatorname{Im} p_{k}$ isomorphe à $Y^{d_{k}}$ (où $d_{k}=\operatorname{dim} \operatorname{Im} \pi_{k}<\infty$ ) est faiblement séquentiellement complète pour tout $k=1,2, \ldots$ 
En ce qui concerne la faible séquentielle complétion de $X \widehat{\widehat{\otimes}} Y$, de la proposition 2, (a), découle, par analogie, le

THÉORÈme 1. Soient $X$ et $Y$ deux espaces de Banach faiblement séquentiellement complets dont l'un possède une décomposition inconditionnelle de dimension finie. Alors le produit tensoriel $X \hat{\otimes} Y$ est faiblement séquentiellement complet si et seulement s'il ne contient aucun sous-espace complémenté isomorphe à $c_{0}$.

Pour des espaces plus généraux mentionnons le résultat de D. R. Lewis [6]: le produit tensoriel $X \hat{\otimes} Y$ de deux espaces $X$ et $Y$ faiblement séquentiellement complets dont l'un possède la propriété d'approximation métrique est faiblement séquentiellement complet si et seulement si tout opérateur linéaire continu de $X^{\prime}$ muni de la topologie $\sigma\left(X^{\prime}, X\right)$ dans $Y$ muni de la topologie $\sigma\left(Y, Y^{\prime}\right)$ est compact.

Pour établir les critères de réflexivité des produits tensoriels nous utilisons le résultat de B. L. Sanders [13]: un espace de Banach avec une décomposition de Schauder $\left(p_{k}\right)$, telle que l'image $\operatorname{Im} p_{k}$ est réflexive pour tout $k=1,2, \ldots$, est réflexif si et seulement si $\left(p_{k}\right)$ est " shrinking " et " boundedly complete"

ThÉorème 2. Soient $X$ et $Y$ deux espaces de Banach réflexifs dont l'un possède une décomposition inconditionnelle de dimension finie. Alors:

(a) Le produit tensoriel $X \hat{\widehat{\otimes}} Y$ est réflexif si et seulement s'il ne contient aucun sous-espace complémenté isomorphe à $c_{0}$.

(b) Le produit tensoriel $X \hat{\otimes} Y$ est réflexif si et seulement s'il ne contient aucun sous-espace complémenté isomorphe à $l_{1}$.

Il faut prouver la partie " si ». Nous supposons que $X$ possède une décomposition inconditionnelle de dimension finie $\left(\pi_{k}\right)$ et soit $\left(p_{k}\right)$ la décomposition de Schauder inconditionnelle correspondante pour $X \hat{\otimes} Y$ ou $X \hat{\otimes} Y$. Comme $Y$ est réflexif, les images $\operatorname{Im} p_{k}$ (étant isomorphes à $Y^{d_{k}}$, $\left.d_{k}=\operatorname{dim} \operatorname{Im} \pi_{k}\right)$ sont réflexives. Comme $X$ est réflexif, $\left(\pi_{k}\right)$ est " shrinking » et "boundedly complete», donc $\left(p_{k}\right)$ est « shrinking " pour $X \hat{\otimes} Y$ et « boundedly complete " pour $X \hat{\otimes} Y$ (cf. Proposition 1). Par conséquent $X \hat{\otimes} Y$ (respectivement $X \hat{\otimes} Y)$ est réflexif si $\left(p_{k}\right)$ est " boundedly complete " (respectivement "shrinking ") et le résultat découle de la Proposition 2, (a) (respectivement (b)).

Remarque. Notons une autre démonstration du Théorème 2. En utilisant le résultat de [6] qui affirme que le produit tensoriel $X \widehat{\otimes} Y$ des espaces réflexifs $X$ 
et $Y$ est réflexif si et seulement s'il est faiblement séquentiellement complet, le Théorème 1 entraîne le (a) du Théorème 2. En utilisant l'identification canonique de $X \hat{\otimes} Y$ à $\left(X^{\prime} \hat{\otimes} Y^{\prime}\right)^{\prime}$, le (a) du Théorème 2 entraîne le (b).

2. Reproductibilité de $l_{q}$ dans $l_{p} \hat{\otimes} Y$.

Nous commençons par deux lemmes simples sur les décompositions de Schauder. Pour une décomposition de Schauder $\left(p_{k}\right)$ notons $P_{n}=\sum_{k=1}^{n} p_{k}$, $n=1,2, \ldots$

LemME 1. Soient $\left(p_{k}\right)$ une décomposition de Schauder d'un espace de Banach $X$ et $1 \leqq q \leqq \infty$. Si $X_{k}=\operatorname{Im} p_{k}, k=1,2, \ldots$, ne contient aucun sous-espace isomorphe $\dot{a}$ $l_{q}$ alors $\operatorname{Im} P_{n}, n=1,2, \ldots$, ne contient aucun sous-espace isomorphe $\grave{a} l_{q}$.

Il est évident que $\operatorname{Im} P_{n}$ est isomorphe à $X_{1} \times X_{2} \times \ldots \times X_{n}$ qui ne contient aucun sous-espace isomorphe à $l_{q}$ puisque $X_{k}$ ne contient aucun sous-espace isomorphe à $l_{q}$ (cf. p. ex. [11]).

Lemme 2. Soient $\left(p_{k}\right)$ une décomposition de Schauder d'un espace de Banach $X$ et $1 \leqq q \leqq \infty$. Si $X$ contient un sous-espace isomorphe à $l_{q}$ et $\operatorname{Im} p_{k}, k=1,2, \ldots$, ne contient aucun sous-espace isomorphe à $l_{q}$ alors il existe une. suite $\left(u_{i}\right)$ de $X$ équivalente à la base canonique de $l_{q}$ et une suite strictement croissante d'entiers $n_{0}<n_{1}<\ldots$ telles que

$$
u_{i}=\left(P_{n_{i}}-P_{n_{i-1}}\right) u_{i}, \quad i=1,2, \ldots
$$

Considérons une suite $\left(x_{i}\right)$ de $X$ équivalente à la base canonique de $l_{q}$ et notons $L\left(x_{i}\right)_{i \geqq m}$ le sous-espace vectoriel engendré par $x_{m}, x_{m+1}, \ldots$ Puisque $\operatorname{Im} P_{n}, n=1,2, \ldots$, ne contient aucun sous-espace isomorphe à $l_{q}$ (cf. Lemme 1), nous avons

(2) $\forall \varepsilon>0, \quad \forall m, \quad \forall n, \quad \exists y \in L\left(x_{i}\right)_{i \geqq m}, \quad\|y\|=1, \quad\left\|P_{n} y\right\|<\varepsilon$.

D'après (2) et le fait que $\lim _{n \rightarrow \infty} P_{n} x=x$ pour tout $x \in X$, nous pouvons construire par récurrence une suite-base bloc normalisée $\left(y_{i}\right)$ de $\left(x_{i}\right)$ et une suite strictement croissante d'entiers $n_{0}=1<n_{1}<n_{2} \ldots$ telles que

$$
\left\|P_{n_{i-1}} y_{i}\right\|+\left\|P_{n_{i}} y_{i}-y_{i}\right\| \leqq \varepsilon / 2^{i}, \quad i=1,2, \ldots .
$$

Comme $\left(y_{i}\right)$ est une suite-base bloc normalisée d'une suite équivalente à la base canonique de $l_{q}$, elle est aussi équivalente à la base canonique de $l_{q}$. Notons

$$
u_{i}=\left(P_{n_{i}}-P_{n_{i-1}}\right) y_{i}, \quad i=1,2, \ldots ;
$$


alors la condition (1) est visiblement satisfaite. Pour $\varepsilon$ suffisamment petit, (3) entraîne l'équivalence de $\left(u_{i}\right)$ à la suite-base normalisée $\left(y_{i}\right)$, donc l'équivalence de $\left(u_{i}\right)$ à la base canonique de $l_{q}$.

Remarque. Nous avons prouvé que si une suite $\left(x_{i}\right)$ de $X$ est équivalente à la base canonique de $l_{q}$ alors $u_{i}=\left(P_{n_{i}}-P_{n_{t-1}}\right) y_{i}$ pour une suite-base bloc normalisée $\left(y_{i}\right)$ de $\left(x_{i}\right)$.

Pour $a=\left(a_{i}\right) \in l_{p}, l \leqq p \leqq \infty$, notons $\|a\|_{p}$ la norme de $a$ dans $l_{p}$. Soient $\sigma \subset\{1,2, \ldots\}$ un ensemble fini d'entiers et $\left(a_{i}\right)_{i \in \sigma}$ une famille de scalaires. Notons $\left\|\left(a_{i}\right)_{i \in \sigma}\right\|_{p}$ la norme dans $l_{p}$ de la suite $\left(b_{i}\right)$ définie par $b_{i}=a_{i}$ si $i \in \sigma$ et $b_{i}=0$ si $i \notin \sigma$.

- Soient $Y$ un espace de Banach, $1 \leqq p \leqq \infty$ et $\left(e_{m}\right)$ la base canonique de $l_{p}$. Notons $\left(\pi_{k}\right)$ la décomposition naturelle de $l_{p}$ définie par

$$
\pi_{k}\left(\sum_{m=1}^{\infty} a_{m} e_{m}\right)=a_{k} e_{k}, \quad k=1,2, \ldots
$$

Alors la décomposition de Schauder $\left(p_{k}\right)$ de $l_{p} \hat{\otimes} Y$ ou $l_{p} \hat{\otimes} Y$ qui lui correspond est inconditionnelle et $\operatorname{Im} p_{k}$ (comme sous-espace de $l_{p} \hat{\otimes} Y$ ou $l_{p} \hat{\otimes} Y$ ) est isomorphe à $Y$ pour tout $k=1,2, \ldots$

Soit

$$
z=\sum_{i=1}^{l}\left(\sum_{m=1}^{\infty} a_{i m} e_{m}\right) \otimes y_{i} \in l_{p} \otimes Y .
$$

En introduisant les applications linéaires $i_{k}: l_{p} \otimes Y \rightarrow Y$ définies par

$$
i_{k} z=\sum_{i=1}^{l} a_{i k} y_{i}
$$

et remarquant que

$$
p_{k} z=\sum_{i=1}^{l} a_{i k} e_{k} \otimes y_{i},
$$

il est immédiat de vérifier (cf. p. ex. [11]) que $\left(g\left(i_{k} p_{k} z\right)\right)_{k=1,2, \ldots} \in l_{p}$ pour tout $g \in Y^{\prime}$ et

$$
\|z\|_{\varepsilon}=\sup \left\{\left\|\sum_{k=1}^{\infty} g\left(i_{k} p_{k} z\right) e_{k}\right\|_{p}: g \in Y^{\prime},\|g\| \leqq 1\right\} .
$$

Pour un ensemble fini d'entiers $\sigma \subset\{1,2, \ldots\}$ et des éléments quelconques $z_{k} \in l_{p} \otimes Y, k \in \sigma$, de (4) découle facilement

$$
\left\|\sum_{k \in \sigma} p_{k} z_{k}\right\|_{\varepsilon}=\sup \left\{\left\|\sum_{k \in \sigma} g\left(i_{k} p_{k} z_{k}\right) e_{k}\right\|_{p}: g \in Y^{\prime},\|g\| \leqq 1\right\},
$$


et d'après (4) et (5) nous avons

$$
\left\|\sum_{k \in \sigma} p_{k} z\right\|_{\varepsilon} \leqq\|z\|_{\varepsilon}, \quad z \in l_{p} \hat{\otimes} Y
$$

THÉORÈmE 3. Soient $Y$ un espace de Banach et $1 \leqq p<q \leqq \infty$. Si $l_{p} \hat{\otimes} Y$ a un sous-espace isomorphe $\dot{a} l_{q}$ alors $Y$ a un sous-espace isomorphe $\dot{a} l_{q}$.

Notons respectivement $\left(p_{k}\right)$ et $\left(q_{k}\right)$ les décompositions de Schauder de $l_{p} \hat{\otimes} Y$ et de $l_{p^{\prime}} \otimes Y^{\prime}$. Pour $n=1,2, \ldots$ notons $P_{n}=\sum_{k=1}^{n} p_{k}$ et $Q_{n}=\sum_{k=1}^{n} q_{k}$.

Supposons que $Y$ ne contienne aucun sous-espace isomorphe à $l_{q}$. Alors, d'après le Lemme 2, il existe une suite strictement croissante d'entiers $n_{0}<n_{1}$ $<\ldots$, une suite $\left(u_{i}\right)$ de $l_{p} \hat{\otimes} Y$ vérifiant (1) et deux réels $0<\lambda \leqq \mu$ tels que pour tout entier $k$ et toute famille $a_{1}, a_{2}, \ldots, a_{k}$ de scalaires

$$
\lambda\left\|\left(a_{i}\right)_{1 \leqq i \leqq k}\right\|_{q} \leqq\left\|\sum_{i=1}^{k} a_{i} u_{i}\right\|_{\pi} \leqq \mu\left\|\left(a_{i}\right)_{1 \leqq i \leqq k}\right\|_{q} .
$$

Rappelons que $l_{p} \hat{\otimes} Y$ s'identifie canoniquement à un sous-espace de $\left(l_{p^{\prime}} \otimes Y^{\prime}\right)^{\prime}$ (cf. [1] ou [11]). On vérifie facilement que

$$
u\left(q_{k} x\right)=\left(p_{k} u\right) x, \quad x \in l_{p^{\prime}} \hat{\otimes} Y^{\prime}, u \in l_{p} \hat{\otimes} Y, k=1,2, \ldots
$$

Fixons $0<\gamma<\lambda$; puisque, d'après (7), $\left\|u_{i}\right\|_{\pi} \geqq \lambda$ pour tout $i=1,2, \ldots$ et

$$
\left\|u_{i}\right\|_{\pi}=\sup \left\{u_{i}(x): x \in l_{p^{\prime}} \otimes_{\varepsilon} Y^{\prime},\|x\|_{\varepsilon} \leqq 1\right\},
$$

nous pouvons choisir une suite $\left(x_{i}\right)$ de $l_{p^{\prime}} \otimes_{\varepsilon} Y^{\prime}$ vérifiant

$$
\begin{aligned}
&\left\|x_{i}\right\|_{\varepsilon} \leqq 1, \quad i=1,2, \ldots, \\
& u_{i}\left(x_{i}\right) \geqq \gamma, \quad i=1,2, \ldots,
\end{aligned}
$$

et quitte à remplacer $x_{i}$ par $\left(Q_{n_{i}}-Q_{n_{i-1}}\right) x_{i}$ nous pouvons supposer que

$$
x_{i}=\left(Q_{n_{i}}-Q_{n_{i-1}}\right) x_{i}, \quad i=1,2, \ldots,
$$

car alors (9) se vérifie par (6) et (10) par (8) et (1). De (1), (11) et (8) découle facilement

$$
i \neq j \Rightarrow u_{i}\left(x_{j}\right)=0, \quad i, j=1,2, \ldots .
$$

Soient maintenant $n$ un entier et $t_{1}, t_{2}, \ldots, t_{n}$ une famille de scalaires. En utilisant (12), (7) et (10) nous obtenons que

$$
\left\|\sum_{j=1}^{n} t_{j} x_{j}\right\|_{\varepsilon} \geqq \sup \left\{\left|u\left(\sum_{j=1}^{n} t_{j} x_{j}\right)\right|: u=\sum_{i=1}^{n} a_{i} u_{i},\|u\|_{\pi} \leqq 1\right\}
$$




$$
\begin{aligned}
& \geqq \sup \left\{\left|\sum_{j=1}^{n} a_{j} t_{j} u_{j}\left(x_{j}\right)\right|:\left\|\left(a_{i}\right)_{1 \leqq i \leqq n}\right\|_{q} \leqq \frac{1}{\mu}\right\} \\
& =\frac{1}{\mu}\left\|\left(t_{j} u_{j}\left(x_{j}\right)\right)_{1 \leqq j \leqq n}\right\|_{q} \geqq \frac{\gamma}{\mu}\left\|\left(t_{j}\right)_{1 \leqq j \leqq n}\right\|_{q^{\prime}} .
\end{aligned}
$$

D'autre part, en utilisant (11) et (5) nous obtenons que

$$
\begin{aligned}
\left\|\sum_{j=1}^{n} t_{j} x_{j}\right\|_{\varepsilon} & =\sup \left\{\left\|\sum_{j=1}^{n} \sum_{k=n_{j-1}+1}^{n_{j}} \varphi\left(i_{k} q_{k} t_{j} x_{j}\right) e_{k}\right\|_{p^{\prime}}: \varphi \in Y^{\prime \prime},\|\varphi\| \leqq 1\right\} \\
& \leqq \begin{cases}\left(\sum_{j=1}^{n}\left|t_{j}\right|^{p^{\prime}}\left(\left\|x_{j}\right\|_{\varepsilon}\right)^{p^{\prime}}\right)^{1 / p^{\prime}} & \text { si } p^{\prime}<\infty, \\
\max _{1 \leqq j \leqq n}\left(\left|t_{j}\right|\left\|x_{j}\right\|_{\varepsilon}\right) & \text { si } p^{\prime}=\infty,\end{cases}
\end{aligned}
$$

donc, d'après (9),

$$
\left\|\sum_{j=1}^{n} t_{j} x_{j}\right\|_{\varepsilon} \leqq\left\|\left(t_{j}\right)_{1 \leqq j \leqq n}\right\|_{p^{\prime}}
$$

ce qui donne avec (13)

$$
\gamma\left\|\left(t_{j}\right)_{1 \leqq j \leqq n}\right\|_{q^{\prime}} \leqq \mu\left\|\left(t_{j}\right)_{1 \leqq j \leqq n}\right\|_{p^{\prime}},
$$

d'où une contradiction puisque $q^{\prime}<p^{\prime}$.

3. Sous-espaces de $l_{p} \hat{\otimes} l_{r}$.

Lemme 3. Soient $1 \leqq p, r \leqq \infty$. Alors $l_{p} \hat{\otimes} l_{r}$ est canoniquement isométriquement isomorphe à un sous-espace de $\left(l_{p^{\prime}} \hat{\otimes} l_{r^{\prime}}\right)^{\prime}$.

D'après [11] $l_{p} \hat{\otimes} l_{r}$ est canoniquement isométriquement isomorphe à un sous-espace de $\left(l_{p^{\prime}} \hat{\otimes}\left(l_{r}\right)^{\prime}\right)^{\prime}$, d'où le résultat pour $r \neq 1$. Pour $r=1$ remarquons que $l_{p} \hat{\otimes} l_{1}$ s'identifie canoniquement à un sous-espace de $\left(l_{p^{\prime}}\right)^{\prime} \hat{\otimes} l_{1}$ qui est canoniquement isométriquement isomorphe à $\left(l_{p^{\prime}} \hat{\otimes} c_{0}\right)^{\prime}$ (cf. [1]).

Soit $\left(p_{k}\right)$ la décomposition de Schauder pour $l_{p} \hat{\otimes} l_{r}$ qui correspond à la décomposition de Schauder naturelle de $l_{p}$; alors $\operatorname{Im} p_{k}, k=1,2, \ldots$, est isomorphe à $l_{r}$. Par l'isométrie naturelle entre $l_{p} \hat{\otimes} l_{r}$ et $l_{r} \hat{\otimes} l_{p}$ nous avons une décomposition de Schauder $\left(r_{k}\right)$ de $l_{p} \hat{\otimes} l_{r}$ qui correspond à la décomposition de Schauder naturelle de $l_{r}$; alors $\operatorname{Im} r_{k}, k=1,2, \ldots$, est isomorphe à $l_{p}$. Notons respectivement $\left(e_{m}\right)$ et $\left(\varepsilon_{m}\right)$ les bases canoniques de $l_{p}$ et $l_{r}$; alors pour

$$
z=\sum_{i=1}^{n}\left(\sum_{m=1}^{\infty} a_{i m} e_{m}\right) \otimes\left(\sum_{m=1}^{\infty} b_{i m} \varepsilon_{m}\right) \in l_{p} \otimes l_{r}
$$

nous avons 


$$
\begin{array}{cc}
p_{k} z=\sum_{i=1}^{n} a_{i k} e_{k} \otimes\left(\sum_{m=1}^{\infty} b_{i m} \varepsilon_{m}\right), & k=1,2, \ldots, \\
r_{l} z=\sum_{i=1}^{n}\left(\sum_{m=1}^{\infty} a_{i m} e_{m}\right) \otimes b_{i l} \varepsilon_{l}, & l=1,2, \ldots,
\end{array}
$$

d'où

$$
p_{k} \circ r_{l}=r_{l} \circ p_{k}, \quad k, l=1,2, \ldots .
$$

Avec les notations $P_{n}=\sum_{k=1}^{n} p_{k}$ et $R_{n}=\sum_{k=1}^{n} r_{k}, n=1,2, \ldots$, nous avons le principal

Lemme 4. Soient $\left(n_{i}\right)$ et $\left(m_{i}\right)$ deux suites strictement croissantes d'entiers et $\left(u_{i}\right)$ une suite normalisée de $l_{p} \hat{\otimes} l_{r}, 1 \leqq p, r \leqq \infty$, telles que

$$
u_{i}=\left(P_{n_{i}}-P_{n_{i-1}}\right) u_{i}=\left(R_{m_{i}}-R_{m_{i-1}}\right) u_{i}, \quad i=1,2, \ldots
$$

Alors le sous-espace fermé $\left[u_{i}\right]_{i \geqq 1}$ de $l_{p} \hat{\otimes} l_{r}$ engendré par $u_{1}, u_{2}, \ldots$ est complémenté dans $l_{p} \hat{\otimes} l_{r}$ et isomorphe à $l_{q}$ où $q=1$ si $p \leqq r^{\prime}$ et $1 / q=1 / p+1 / r$ (avec $1 / \infty=0)$ si $p>r^{\prime}$.

Remarquons que $\left(u_{i}\right)$ est une suite-base inconditionnelle de $l_{p} \hat{\otimes} l_{r}$ car

$$
u_{i}=\left(P_{n_{i}}-P_{n_{i-1}}\right) u_{i} \neq 0
$$

et la décomposition $\left(p_{k}\right)$ de $l_{p} \hat{\otimes} l_{r}$ est inconditionnelle.

Soient $\left(q_{k}\right)$ et $\left(s_{k}\right)$ les décompositions de Schauder de $l_{p^{\prime}} \hat{\hat{\otimes}} l_{r^{\prime}}$ définies respectivement de la même façon que $\left(p_{k}\right)$ et $\left(r_{k}\right)$ pour $l_{p} \hat{\otimes} l_{r}$. Par analogie à (14) nous avons

$$
q_{k} \circ s_{l}=s_{l} \circ q_{k}, \quad k, l=1,2, \ldots .
$$

En identifiant canoniquement $l_{p} \hat{\otimes} l_{r}$ à un sous-espace de $\left(l_{p^{\prime}} \hat{\otimes} l_{r^{\prime}}\right)^{\prime}$ (cf. Lemme 3) nous avons pour $u \in l_{p} \hat{\otimes} l_{r}, x \in l_{p^{\prime}} \hat{\otimes} l_{r^{\prime}}$ et $k=1,2, \ldots$

$$
\left(p_{k} u\right) x=u\left(q_{k} x\right), \quad\left(r_{k} u\right) x=u\left(s_{k} x\right) .
$$

Notons $Q_{n}=\sum_{k=1}^{n} q_{k}$ et $S_{n}=\sum_{k=1}^{n} s_{k}, \quad n=1,2, \ldots$ Comme dans la démonstration du Théorème 3 , nous pouvons trouver une suite $\left(x_{i}\right)$ de $l_{p^{\prime}} \hat{\otimes} l_{r^{\prime}}$ vérifiant $(9)$ et $(10)($ avec $0<\gamma<1)$. Quitte à remplacer $x_{i}$ par

$$
\left(Q_{n_{i}}-Q_{n_{i-1}}\right)\left(S_{m_{i}}-S_{m_{i-1}}\right) x_{i}=\left(S_{m_{i}}-S_{m_{i-1}}\right)\left(Q_{n_{i}}-Q_{n_{i-1}}\right) x_{i}
$$

(cf. (16)) nous pouvons supposer que

$$
x_{i}=\left(Q_{n_{i}}-Q_{n_{i-1}}\right) x_{i}=\left(S_{m_{i}}-S_{m_{i-1}}\right) x_{i}, \quad i=1,2, \ldots,
$$

car alors (9) se vérifie par (6) et (10) par (17) et (15). De (15), (18) et (17) 
découle facilement (12). D'après (9) et (10) nous pouvons supposer la suite $\left(x_{i}\right)$ normalisée et vérifiant (10).

Il est établi dans [11] (cf. la démonstration du Théorème du 5) qu'une suite normalisée $\left(x_{i}\right)$ de $l_{p^{\prime}} \hat{\otimes} l_{r^{\prime}}$ vérifiant (18) est isométriquement équivalente à la base canonique de $l_{q^{\prime}}$ (il est élémentaire de vérifier que $Q_{n}$ et $S_{n}$ s'identifient par l'isométrie de $l_{p^{\prime}} \hat{\otimes} l_{r^{\prime}}$ dans $s l_{r^{\prime}}\left(l_{p^{\prime}}\right)$ de [11] respectivement à $\tilde{Q}_{n}$ et à $P_{n}$ de [11]).

En reprenant la démonstration du Lemme de [12] et utilisant (15), (17), (18), (10), $\left\|u_{i}\right\|=1, i=1,2, \ldots$, et (12) nous pouvons montrer que l'application $P$ : $l_{p^{\prime}} \hat{\otimes} l_{r^{\prime}} \rightarrow l_{p^{\prime}} \hat{\otimes} l_{r^{\prime}}$ définie par

$$
P x=\sum_{j=1}^{\infty} \varphi_{j}(x) x_{j}, \quad \text { où } \quad \varphi_{j}=\frac{u_{j}}{u_{j}\left(x_{j}\right)},
$$

est une projection continue de $l_{p^{\prime}} \hat{\otimes} l_{r^{\prime}}$ sur $\left[x_{i}\right]_{i \geqq 1}$.

Donc $P^{\prime}$ est une projection continue de $\left(l_{p^{\prime}} \otimes l_{r^{\prime}}\right)^{\prime}$ avec $\operatorname{Im} P^{\prime}$ isomorphe à $\left(l_{q^{\prime}}\right)^{\prime}$.

Pour $i=1,2, \ldots$ et $x \in l_{p^{\prime}} \hat{\hat{\otimes}} l_{\boldsymbol{r}^{\prime}}$ en utilisant (12) nous avons

$$
\left(P^{\prime} u_{i}\right) x=\sum_{j=1}^{\infty} \varphi_{j}(x) u_{i}\left(x_{j}\right)=u_{i}(x)
$$

d'où

$$
\left[u_{i}\right]_{i \geqq 1} \subset \operatorname{Im} P^{\prime} .
$$

Notons que les sous-espaces fermés engendrés par $u_{1}, u_{2}, \ldots$ dans $l_{p} \hat{\otimes} l_{r}$ et $\left(l_{p^{\prime}}, \hat{\otimes} l_{r^{\prime}}\right)^{\prime}$ coïncident puisque $l_{p} \hat{\otimes} l_{r}$ est fermé dans $\left(l_{p^{\prime}}, \hat{\hat{\otimes}} l_{r^{\prime}}\right)^{\prime}$.

Nous allons considérer deux éventualités:

1) $p=r=\infty$ et

2) $p \neq \infty$ ou $r \neq \infty$.

1) Dans ce cas $q=\infty, q^{\prime}=1$ et

$$
\|P x\|_{\varepsilon}=\sum_{j=1}^{\infty}\left|\varphi_{j}(x)\right|, \quad x \in l_{p^{\prime}} \hat{\otimes} l_{r^{\prime}}=l_{1} \hat{\otimes} l_{1} .
$$

Comme $\left(u_{i}\right)$ est une suite-base normalisée, il existe un réel $\alpha>0$ tel que pour tout entier $n$ et toute famille $a_{1}, a_{2}, \ldots, a_{n}$ de scalaires

$$
\alpha \max _{1 \leqq i \leqq n}\left|a_{i}\right| \leqq\left\|\sum_{i=1}^{n} a_{i} u_{i}\right\|_{\pi} .
$$

D'autre part, pour tout $x \in l_{1} \hat{\otimes} l_{1}$ nous avons en utilisant (19) et (12) 


$$
\begin{aligned}
\left|\left(\sum_{i=1}^{n} a_{i} u_{i}\right)(x)\right| & =\left|\sum_{i=1}^{n} a_{i} \varphi_{i}(x) u_{i}\left(x_{i}\right)\right| \leqq \max _{1 \leqq i \leqq n}\left|a_{i}\right| \sum_{i=1}^{n}\left|\varphi_{i}(x)\right| \\
& \leqq \max _{1 \leqq i \leqq n}\left|a_{i}\right|\|P x\|_{\varepsilon} \leqq\|P\| \max _{1 \leqq i \leqq n}\left|a_{i}\right|\|x\|_{\varepsilon}
\end{aligned}
$$

d'où

$$
\left\|\sum_{i=1}^{n} a_{i} u_{i}\right\|_{\pi} \leqq\|P\| \max _{1 \leqq i \leqq n}\left|a_{i}\right| .
$$

Par conséquent le sous-espace $\left[u_{i}\right]_{i \geqq 1}$ de $c_{0} \hat{\otimes} c_{0}$ est isomorphe à $c_{0}$. Il est complémenté dans $c_{0} \hat{\otimes} c_{0}$ puisque $c_{0} \hat{\otimes} c_{0}$ est séparable.

2) Dans ce cas $q \neq \infty, q^{\prime} \neq 1,\left(l_{q^{\prime}}\right)^{\prime}=l_{q}$ et $\operatorname{Im} P^{\prime}$ est donc isomorphe à $l_{q}$. Il suffit de démontrer que $\left[u_{i}\right]_{i \geqq 1}=\operatorname{Im} P^{\prime}$ car alors la restriction de $P^{\prime}$ sur $l_{p} \hat{\otimes} l_{r}$ est une projection continue de $l_{p} \hat{\otimes} l_{r}$ sur $\left[u_{i}\right]_{i \geqq 1}$. Pour cela il nous reste à montrer que Im $P^{\prime} \subset\left[u_{i}\right]_{i \geqq 1}$. Remarquons que la suite-base $\left(u_{i}\right)$ de $l_{p} \hat{\otimes} l_{r}$ est « boundedly complete " puisqu'elle est inconditionnelle et $\left[u_{i}\right]_{i \geqq 1}$, étant isomorphe à un sous-espace de $l_{q}$, ne contient aucun sous-espace isomorphe à $c_{0}$.

Fixons $u=P^{\prime} u \in\left(l_{p^{\prime}} \hat{\otimes} l_{r^{\prime}}\right)^{\prime}$. Pour tout $x \in l_{p^{\prime}} \stackrel{\otimes}{\otimes} l_{r^{\prime}}$ nous avons

$$
u(x)=\lim _{n \rightarrow \infty}\left(\sum_{j=1}^{n} \frac{u\left(x_{j}\right)}{u_{j}\left(x_{j}\right)} u_{j}\right)(x)=\lim _{n \rightarrow \infty} v_{n}(x)
$$

ce qui montre que la suite $\left(v_{n}\right)$ est bornée dans $\left(l_{p^{\prime}} \hat{\otimes} l_{r^{\prime}}\right)^{\prime}$ et donc aussi dans $l_{p} \hat{\otimes} l_{r}$. La suite-base $\left(u_{i}\right)$ étant " boundedly complete ", la suite $\left(v_{n}\right)$ converge dans $l_{p} \hat{\otimes} l_{r}$ et par conséquent sa limite $u \in\left[u_{i}\right]_{i \geqq 1}$ ce qui achève la démonstration.

THÉORÈmE 4. Soient $1 \leqq p, q, r \leqq \infty$. Si le produit tensoriel $l_{p} \hat{\otimes} l_{r}$ contient un sous-espace isomorphe $\grave{a} l_{q}$ alors $q=p$ ou $q=r$ ou $q=s$ où $s=1$ si $p \leqq r^{\prime}$ et $1 / s$ $=1 / p+1 / r($ avec $1 / \infty=0)$ si $p>r^{\prime}$.

Conservons les notation du Lemme 4. Supposons que $l_{q}$ soit isomorphe à un sous-espace de $l_{p} \hat{\otimes} l_{r}$ et que $q \neq p$ et $q \neq r$. Comme $q \neq r$, d'après le Lemme 2 , il existe une suite-base $\left(z_{i}\right)$ de $l_{p} \hat{\otimes} l_{r}$ équivalente à la base canonique de $l_{q}$ et une suite strictement croissante d'entiers $n_{0}<n_{1}<\ldots$ telles que

$$
z_{i}=\left(P_{n_{i}}-P_{n_{i-1}}\right) z_{i}, \quad i=1,2, \ldots
$$

Comme $q \neq p$, d'après le Lemme 2 et la Remarque au Lemme 2 , il existe une suite-base bloc normalisée $\left(y_{i}\right)$ de $\left(z_{i}\right)$ et une suite strictement croissante d'entiers $M_{0}<M_{1}<\ldots$ telles que la suite

$$
u_{i}=\left(R_{M_{i}}-R_{M_{i-1}}\right) y_{i}
$$


est équivalente à la base canonique de $l_{q}$. Soit la suite $\left(y_{i}\right)$ de la forme

$$
y_{i}=\sum_{j=p_{t-1}+1}^{p_{i}} \lambda_{j} z_{j}
$$

notons $N_{i}=n_{p_{i}}$. Alors

$$
\left(P_{N_{i}}-P_{N_{i-1}}\right) y_{i}=\sum_{j=p_{t-1}+1}^{p_{i}} \lambda_{j}\left(P_{N_{i}}-P_{N_{i-1}}\right)\left(P_{n_{j}}-P_{n_{j-1}}\right) z_{j}=y_{i}
$$

et donc, en utilisant (14),

$$
\left(P_{N_{i}}-P_{N_{i-1}}\right) u_{i}=\left(R_{M_{i}}-R_{M_{t-1}}\right)\left(P_{N_{i}}-P_{N_{i-1}}\right) y_{i}=u_{i} .
$$

En définitive, nous avons trouvé une suite $\left(u_{i}\right)$ de $l_{p} \hat{\otimes} l_{r}$ équivalente à la base canonique de $l_{q}$ et vérifiant (15). Nous concluons en utilisant le Lemme 4.

Par la méthode de la démonstration du Théorème de [12] du Lemme 4 découle le

THÉORÈME 5. Soit $X$ un sous-espace fermé de dimension infinie de $l_{p} \hat{\otimes} l_{r}$. Alors $X$ contient un sous-espace complémenté dans $l_{p} \hat{\otimes} l_{r}$ isomorphe à $l_{q}$ avec $q=p$ ou $q=r$ ou $q=s$ où $s=1$ si $p \leqq r^{\prime}$ et $1 / s=1 / p+1 / r$ (avec $\left.1 / \infty=0\right)$ si $p>r^{\prime}$.

\section{BIBLIOGRAPHIE}

1. A. Grothendieck, Produits tensoriels topologiques et espaces nucléaires, Mem. Amer. Math. Soc. 16 (1955).

2. S. Heinrich, The weak sequential completeness of Banach operator ideals, Sibirsk. Mat. Ž. 17 (1976), 1160-1167 (Russian). (English translation: Siberian Math. J. 17 (1976), 857-862.)

3. J. R. Holub, Hilbertian operators and reflexive tensor products, Pacific J. Math. 36 (1971), 185194.

4. N. J. Kalton, Schauder decompositions in locally convex spaces, Proc. Cambridge Philos. Soc. 68 (1970), 377-392.

5. N. J. Kalton, Spaces of compact operators, Math. Ann. 208 (1974), 267-278.

6. D. R. Lewis, Conditional weak compactness in certain inductive tensor products, Math. Ann. 201 (1973), 201-209.

7. J. Lindenstrauss and L. Tzafriri, Classical Banach spaces I (Ergebnisse der Mathematik und Ihrer Grenzgebiete 92), Springer-Verlag, Berlin - Heidelberg - New York, 1977.

8. E. Oja, Unconditional Schauder decompositions in locally convex spaces, (Russian. Estonian and English summaries) Tartu Riikl. Ül. Toimetised 374 (1975), 90-116.

9. E. Oja, Unconditional Schauder decompositions, and semi-reflexive spaces, (Russian. Estonian and English summaries) Tartu Riikl. Ül. Toimetised 431 (1977), 82-97.

10. W. H. Ruckle, The infinite sum of closed subspaces of an F-space, Duke Math. J. 31 (1964), 543-554. 
11. C. Samuel, Sur la reproductibilité des espaces $l_{p}$, Math. Scand. 45 (1979), 103-117.

12. C. Samuel, Sur les sous-espaces de $l_{p} \otimes l_{q}$, Math. Scand. 47 (1980), 247-250.

13. B. L. Sanders, Decompositions and reflexivity in Banach spaces, Proc. Amer. Math. Soc. 16 (1965), 204-208.

FACULTÉ DE MATHÉMATIQUES UNIVERSITÉ DE TARTU

46, VANEMUISE

202400 TARTU

URSS
DÉPARTEMENT DE MATHÉMATIQUE-INFORMATIQUE FACULTÉ DES SCIENCES DE LUMINY

70, ROUTE LÉON-LACHAMP

13288 MARSEILLE CEDEX 9

FRANCE 